\title{
Collagen type II is downregulated in the degenerative nucleus pulposus and contributes to the degeneration and apoptosis of human nucleus pulposus cells
}

\author{
CHENGJIE LIAN $^{1 *}$, BO GAO $^{1 *}$, ZIZHAO WU $^{1}$, XIANJIAN QIU $^{1}$, YAN PENG $^{1}$, \\ ANJING LIANG $^{1}$, CAIXIA XU ${ }^{2}$, PEIQIANG $\mathrm{SU}^{3}$ and DONGSHENG HUANG ${ }^{1}$ \\ ${ }^{1}$ Department of Orthopedics, Sun Yat-sen Memorial Hospital of Sun Yat-sen University, Guangzhou, Guangdong 510120; \\ ${ }^{2}$ Research Centre for Translational Medicine; ${ }^{3}$ Department of Orthopedics, The First Affiliated Hospital of \\ Sun Yat-sen University, Guangzhou, Guangdong 510080, P.R. China
}

Received October 24, 2016; Accepted June 12, 2017

DOI: $10.3892 / \mathrm{mmr} .2017 .7178$

\begin{abstract}
Degenerative disc disease (DDD) is a common degenerative condition initiated mainly within the nucleus pulposus (NP). To date, the etiopathogenesis of DDD remains unclear, and because no effective therapeutic strategies are available to target its pathological processes, DDD is still treated with symptomatic interventions that are far from adequate. Collagen type II is one of the major matrix components of the NP, and is considered to be essential to NP homeostasis. However, the specific mechanisms by which collagen type II influences NP cells remain unknown. In the present study, collagen type II expression was detected using immunohistochemistry analysis and quantitative polymerase chain reaction, and it was demonstrated to be significantly downregulated in NP tissues from patients with DDD compared with nondegenerative controls. To further explore the mechanism in vitro, interleukin (IL)- $1 \beta$ stimulation was used to induce degeneration of a human NP cell line. IL-1 $\beta$ stimulation upregulated both the mRNA and protein levels of the catabolic markers matrix metalloproteinase 13 (MMP13) and a disintegrin and metalloproteinase with thrombospondin motifs 4 (ADAMTS4), while it downregulated the anabolic
\end{abstract}

Correspondence to: Professor Dongsheng Huang, Department of Orthopedics, Sun Yat-sen Memorial Hospital of Sun Yat-sen University, 107 West Yan Jiang Road, Guangzhou, Guangdong 510120, P.R. China

E-mail: huangdongshen18@hotmail.com

Professor Peiqiang Su, Department of Orthopedics, The First Affiliated Hospital of Sun Yat-sen University, 58 Zhongshan Road, Guangzhou, Guangdong 510080, P.R. China

E-mail:supq@mail.sysu.edu.cn

*Contributed equally

Key words: degenerative disc disease, collagen type II, nucleus pulposus cell, apoptosis makers aggrecan and collagen type II. However, addition of purified collagen type II prevented this IL-1 $\beta$-induced metabolic disturbance of the NP cells. Furthermore, IL-1 $\beta$ stimulation significantly promoted apoptosis in NP cells, while collagen type II treatment decreased the apoptotic rate and the protein levels of cleaved caspase-3. In conclusion, collagen type II exhibited protective effects in suppressing NP cell degeneration through its anticatabolic, proanabolic and antiapoptotic effects, suggesting that it may be a promising therapeutic agent for the prevention and treatment of DDD.

\section{Introduction}

Low back pain is a serious global health problem that imposes immense social and financial burdens $(1,2)$, and degenerative disc disease (DDD) is a major cause in $\sim 40 \%$ of DDD cases (3). The etiopathogenesis of DDD remains unclear; it is thought to involve multiple factors, including imbalances of anabolism and catabolism of the extracellular matrix (ECM), increased inflammatory cytokines, and excessive apoptosis of nucleus pulposus (NP) cells (4-6). During NP degeneration, significantly upregulated proinflammatory cytokines, interleukin (IL)-1 $\beta$ and tumor necrosis factor (TNF) $\alpha$, drive the expression of various matrix metalloproteinases (MMPs) and aggrecanases, such as a disintegrin and metalloproteinase with thrombospondin motifs (ADAMTS). Thereafter these catabolic proteases can initiate proteolytic degradation of ECM macromolecules (mainly collagen type II and proteoglycans) and induce NP cell apoptosis, resulting in progressive deterioration of the intervertebral discs (IVDs) (4-8). Because no effective therapeutic strategies are available to target these key pathological processes, DDD is still treated with symptomatic interventions that are predominantly inadequate (9).

Collagen type II is encoded by the collagen type II $\alpha 1$ (COL2A1) gene, and mainly synthesized by chondrocytes and NP cells (10). Collagen type II, which is one of the major matrix components of the NP, can form an irregular network to hold proteoglycans and water together and maintain the structural stability of NP tissue (3). Both the decreased secretion of collagen type II and the increased breakdown 
of ECM collagen fibers are regarded as typical pathological changes in DDD $(4,9)$. In addition, the Col2a1-null mouse has been reported to be unable to remove the notochord and develop normal IVDs, which further confirms the indispensability of collagen type II for NP development and homeostasis (11). Of note, collagen type II, which was initially regarded as a structural matrix molecule, is now thought to also function as an extracellular signaling molecule. Studies in chondrocytes reveal that collagen type II can modulate chondrocyte proliferation, differentiation, and metabolism through complex signaling pathways (12-14). However, the role of collagen type II in the biological functions of NP cells remains unknown.

The present study demonstrated that collagen type II is downregulated in NP tissues of patients with DDD, and treatment of NP cells in vitro with purified collagen type II prevented NP cell degeneration via its anticatabolic, proanabolic, and antiapoptotic effects.

\section{Materials and methods}

Ethics statement. The present study was approved by the Institutional Research Ethical Committee of Sun Yat-sen University (Guangzhou, China). Written informed consent was obtained from all subjects participating in the study.

Antibodies and reagents. The following primary antibodies (Abs) were purchased from Cell Signaling Technology, Inc. (Danvers, MA, USA): caspase-3 (cat. no. 9662) and cleaved caspase-3 (c-caspase-3) (cat. no. 9661). Abs against GAPDH (cat. no. ab8245), COL2A1 (cat. no. ab34712), matrix metalloproteinase (MMP) 13 (cat. no. ab39012), a disintegrin and metalloproteinase with thrombospondin motifs 4 (ADAMTS4; cat. no. ab28285), aggrecan (ACAN; cat. no. ab36861), and $\alpha$-tubulin (cat. no. ab7291) were obtained from Abcam (Cambridge, Cambridgeshire, UK). Horseradish peroxidase (HRP)-conjugated goat anti-rabbit immunoglobulin (Ig) G and goat anti-mouse IgG secondary antibodies (cat. nos. 31460 and 31430, respectively) were purchased from Thermo Fisher Scientific, Inc. (Waltham, MA, USA). Recombinant human IL-1 $\beta$ was purchased from R\&D Systems, Inc. (Minneapolis, MN, USA). Purified collagen type II was purchased from Chondrex, Inc. (Redmond, WA, USA).

Culture and treatment of human NP cells. Human NP cells were obtained from ScienCell (Carlsbad, CA, USA) and cultured in Human NP Cell medium (ScienCell) at $37^{\circ} \mathrm{C} / 5 \% \mathrm{CO}_{2}$. The medium was changed every 3 days. When the cells reached $80-90 \%$ confluence, they were trypsinized, counted, and plated again at a density of $1.5 \times 10^{6}$ cells $/ \mathrm{ml}$. A total of $12 \mathrm{~h}$ after plating, cells were treated with or without $10 \mathrm{ng} / \mathrm{ml} \mathrm{IL-1 \beta}$, $100 \mu \mathrm{g} / \mathrm{ml}$ purified collagen type II, and/or vehicle $(0.05 \mathrm{M}$ acetic acid, as used for collagen type II dissolution) for $48 \mathrm{~h}$ and then used in subsequent experiments.

Reverse transcription-quantitative polymerase chain reaction $(R T-q P C R)$. Total RNA from cells and tissue samples was extracted using the RNeasy mini kit (Qiagen GmbH, Hilden, Germany) and converted to cDNA using PrimeScript RT Master Mix (Takara Bio, Inc., Otsu, Japan). qPCR was performed on a LightCycler 480 Real-Time PCR Detection System (Roche Diagnostics, Basel, Kanton Basel-Stadt, Switzerland) using SYBR Green I Master Mix (Roche Diagnostics). The thermocycling conditions were: $95^{\circ} \mathrm{C}$ for $5 \mathrm{~min}$, followed by 40 cycles of $95^{\circ} \mathrm{C}$ for $10 \mathrm{sec}, 60^{\circ} \mathrm{C}$ for $20 \mathrm{sec}$ and $72^{\circ} \mathrm{C}$ for $20 \mathrm{sec}$. Expression of COL2A1, MMP13, ADAMTS4, and ACAN were examined. The expression level of the GAPDH gene was used as reference. Primer sequences are listed in Table I. All primers were synthesized by Invitrogen (Thermo Fisher Scientific, Inc.). Each PCR reaction was performed in triplicate. The $\mathrm{Cq}$ value of the GAPDH was subtracted from the $\mathrm{Cq}$ value of the target gene $(\Delta \mathrm{Cq})$ and the average $\Delta \mathrm{Cq}$ value of the triplicates was recorded. The relative expression levels of each gene were determined using the $2^{-\Delta \Delta \mathrm{Cq}}$ method (15).

Western blotting. Total protein was extracted using the radioimmunoprecipitation assay lysis buffer (Beyotime Institute of Biotechnology, Shanghai, China). Equal amounts (60 $\mu \mathrm{g})$ of each sample were subjected to SDS-PAGE on a $10 \%$ gel and transferred to polyvinylidene difluoride membranes (EMD Millipore, Billerica, MA, USA). Membranes were blocked with $5 \%$ non-fat dry milk for $1 \mathrm{~h}$ at room temperature and then incubated with anti-GAPDH $(1: 5,000)$, anti-caspase-3 (1:1,000), anti-c-caspase-3 (1:1,000), anti-COL2A1 (1:1,000), anti-MMP13 (1:2,000), anti-ADAMTS4 (1:2,000), anti-ACAN (1:500), and anti- $\alpha$-tubulin $(1: 5,000)$ antibodies at $4^{\circ} \mathrm{C}$ overnight. Then membranes were incubated with the appropriate HRP-conjugated secondary antibody $(1: 2,000)$ for $1 \mathrm{~h}$ at room temperature and signals were visualized using an enhanced chemiluminescence kit (EMD Millipore).

Human NP tissues. Human lumbar NP tissues were collected from 15 patients with DDD (4 males and 11 females; mean age, $56.9 \pm 8.3$ years) and 8 patients with idiopathic scoliosis ( 8 females; mean age, $14.3 \pm 2.7$ years) which served as nondegenerative controls $(16,17)$. Magnetic resonance imaging (MRI) was performed in all patients prior to surgery, and disc degeneration was assessed using Pfirrmann's grading system based on T2-weighted MRI images (18). DDD patients were classified as grade IV-V, while idiopathic scoliosis patients were classified as grade I-II. All patients underwent surgical treatment at Sun Yat-sen Memorial Hospital of Sun Yat-sen University (Guangzhou, China) or The First Affiliated Hospital of Sun Yat-sen University (Guangzhou, China) from January 2013 to December 2015, during which NP tissues were collected.

Immunohistochemical (IHC) analysis. Paraformaldehyde (4\%) was used for fixation of the tissues at room temperature for $1 \mathrm{~h}$. Paraffin sections (4- $\mu \mathrm{m}$ thick) were prepared and $5 \%$ bovine serum albumin (Sigma-Aldrich; Merck KGaA, Darmstadt, Germany) was used as the blocking reagent for $20 \mathrm{~min}$ at room temperature. IHC was performed using the Histostain-Plus kit (Origin Technologies, Inc., Beijing, China) with the anti-COL2A1 antibody (Abcam, 1:500) at $4^{\circ} \mathrm{C}$ overnight. Detection was conducted using a DAB Horseradish Peroxidase Color Development kit (Origin Technologies, Inc.). Semi-quantitative analysis of IHC images through integrated optical density (IOD) was performed using Image-Pro Plus 7 software (Media Cybernetics, Inc., Rockville, MD, USA). 
Terminal deoxynucleotidyl-transferase-mediated dUTP nick end labelling (TUNEL) assay. A TUNEL assay was performed using the MEBSTAIN Apoptosis TUNEL kit Direct (MBL International Co., Woburn, MA, USA), according to the manufacturer's instructions. The \% of TUNEL-positive cells relative to propidium iodide (PI)-stained cells was calculated in 5 random fields by microscopy for each sample. Three independent experiments were conducted and quantified for each experimental group.

Flow cytometry analysis of cell apoptosis. NP cells were plated on 24 -well plates at $1 \times 10^{5}$ cells/well. Following $12 \mathrm{~h}$, NP cells were treated with $10 \mathrm{ng} / \mathrm{ml} \mathrm{IL-1} \beta$ for $48 \mathrm{~h}$ to induce apoptosis. Purified collagen type II $(100 \mu \mathrm{g} / \mathrm{ml})$ was added simultaneously to inhibit the effect of IL-1 $\beta$. To distinguish non-apoptotic cells from cells in early apoptosis and late apoptosis/necrosis, cells were stained with the fluorescein isothiocyanate (FITC)-Annexin V Apoptosis Detection kit with 7-aminoactinomycin D (7-AAD) according to the manufacturer's instructions (Sony Biotechnology Inc., San Jose, CA, USA) and then analyzed with a Gallios Flow Cytometer (Beckman Coulter, Inc., Brea, CA, USA).

Statistical analysis. All quantitative data were presented as means \pm standard deviation. Statistical analysis of the results from the clinical samples was performed using the Mann-Whitney U test. Statistical analysis of the in vitro results was performed using one-way analysis of variance followed by Dunnett's post hoc test. All statistical analyses were conducted with SPSS 13.0 statistical software (SPSS, Inc., Chicago, IL, USA). $\mathrm{P}<0.05$ was considered to indicate a statistically significant difference.

\section{Results}

Expression of collagen type II is downregulated in degenerated human NP tissues. To determine whether the expression of collagen type II was altered in degenerated human NP tissues, 15 sampled from degenerative discs and 8 samples from nondegenerative discs were selected. IHC and RT-qPCR were conducted to assess the expression level of collagen type II in NP tissues. Compared with the nondegenerative controls, collagen type II expression was significantly decreased in degenerated NP tissues at both the mRNA and protein level (Fig. 1).

Collagen type II treatment protects human NP cells from IL-1 $\beta$-induced metabolic disturbance. To determine whether collagen type II may affect NP cell degeneration, human NP cells were treated in vitro with or without vehicle, $10 \mathrm{ng} / \mathrm{ml}$ IL-1 $\beta$ and $/$ or $100 \mu \mathrm{g} / \mathrm{ml}$ purified collagen type II. RT-qPCR and western blotting revealed that IL-1 $\beta$ treatment alone significantly upregulated the expression levels of catabolic genes MMP13 and ADAMTS4, while it downregulated the expression of the NP-specific markers COL2A1 and ACAN, compared with control vehicle-treated cells (Fig. 2). Following addition of purified collagen type II to the IL- $1 \beta$ treatment, the effects of IL-1 $\beta$ on MMP13, ADAMTS4, CLO2A1 and ACAN mRNA and protein expression levels were significantly reversed (Fig. 2). These results demonstrated that collagen
Table I. Primers used for reverse transcription-quantitative polymerase chain reaction.

\begin{tabular}{lll}
\hline Gene & Primer & \multicolumn{1}{c}{ Sequence (5'-3') } \\
\hline GAPDH & Forward & AGAAAAACCTGCCAAATATGAT \\
& GAC \\
& Reverse & TGGGTGTCGCTGTTGAAGTC \\
COL2A1 & Forward & GGCAATAGCAGGTTCACGTACA \\
& Reverse & CGATAACAGTCTTGCCCACTT \\
ACAN & Forward & TGCATTCCACGAAGCTAACCTT \\
& Reverse & GACGCCTCGCCTTCTTGAA \\
ADAMTS4 & Forward & GAGGAGGAGATCGTGTTCCA \\
& Reverse & CCAGCTCTAGTAGCAGCGTC \\
MMP13 & Forward & CCAGACTTCACGATGGCATTG \\
& Reverse & GGCATCTCCTCCATAATTTGC \\
\hline
\end{tabular}

COL2A1, collagen type II $\alpha 1$; ACAN, aggrecan; ADAMTS4, a disintegrin and metalloproteinase with thrombospondin motifs 4; MMP13, matrix metalloproteinase 13 .

type II protected NP cells from IL-1 $\beta$-induced metabolic disturbance.

Collagen type II treatment inhibits IL-1 $\beta$-induced apoptosis in human NP cells. To confirm whether collagen type II may modify NP cell apoptosis, human NP cells were treated in vitro with or without vehicle, $10 \mathrm{ng} / \mathrm{ml} \mathrm{IL-1 \beta}$ and/or $100 \mu \mathrm{g} / \mathrm{ml}$ purified collagen type II. Annexin V and 7-AAD staining was performed to assess cell apoptosis. IL-1 $\beta$ treatment increased the $\%$ of apoptotic cells in the total cell population (measured as the sum of both early and late-stage apoptotic cells) from $7.52 \pm 0.84$ to $27.06 \pm 3.58 \%$ in the control and the IL1 $\beta$-treated cells respectively (Fig. 3A). Addition of collagen type II to IL-1 $\beta$ treatment significantly decreased the apoptotic rates to $14.52 \pm 2.86 \%$ (Fig. 3A). Similar results were obtained by TUNEL assay, with IL-1 $\beta$ treatment increasing the $\%$ of TUNEL-positive cells compared to control, and addition of collagen type II significantly reversing this effect (Fig. 3B). Furthermore, because caspase-3 is a critical mediator of apoptosis, the protein levels of caspase- 3 and cleaved caspase- 3 (which represents the active form of caspase-3) were detected by western blot analysis. The upregulated cleaved caspase- 3 levels mediated by IL- $1 \beta$ treatment were suppressed by the addition of purified collagen type II (Fig. 3C). These results demonstrated that collagen type II had a protective effect against IL-1 $1 \beta$-induced NP cell apoptosis.

\section{Discussion}

DDD is a chronic degenerative condition initiated mainly within the NP; genetic predisposition, environmental influence, and aging contribute to its pathogenesis $(3,4,7,9,19)$. Loss of NP cells and destruction of the normal ECM are believed to have important roles in the progression of NP degeneration $(5,6,20,21)$. The present study demonstrated that collagen type II is significantly decreased in the NP tissues of patients with DDD, and that addition of purified collagen type II exerted anticatabolic, proanabolic and antiapoptotic 
A

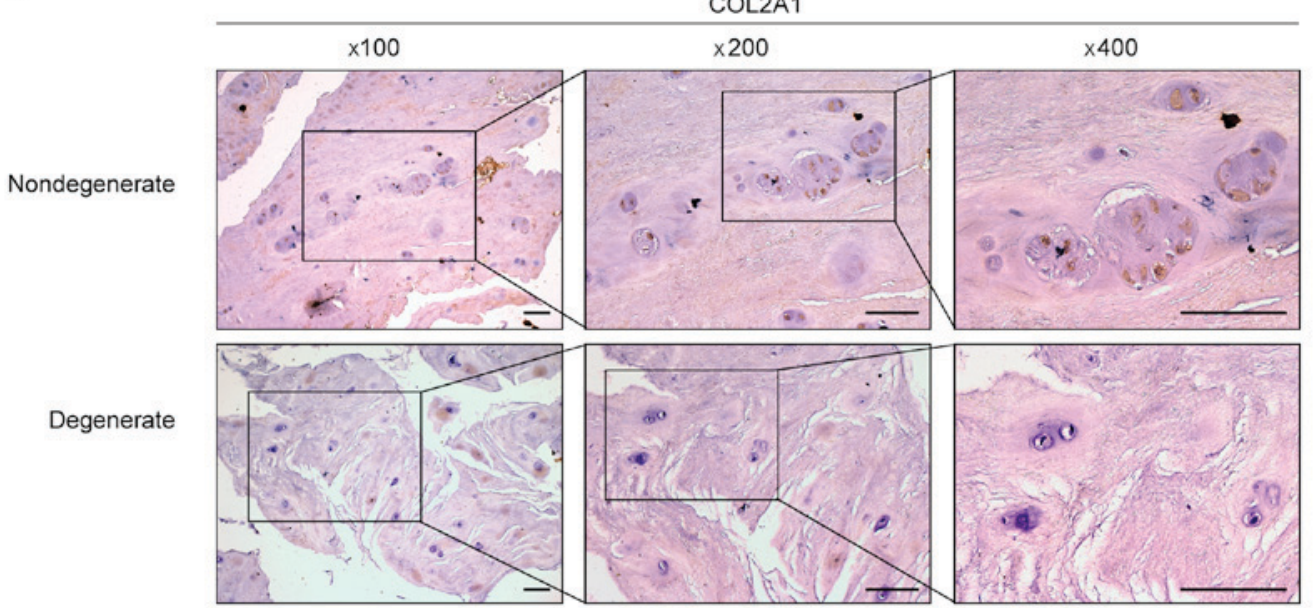

B

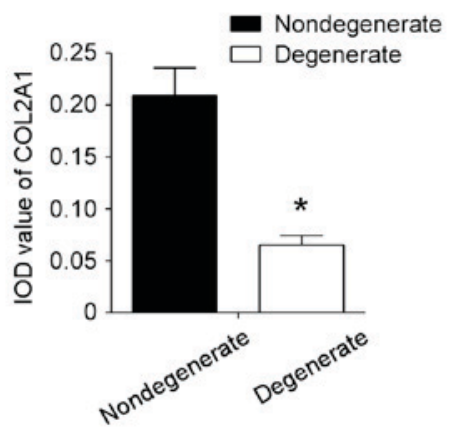

C

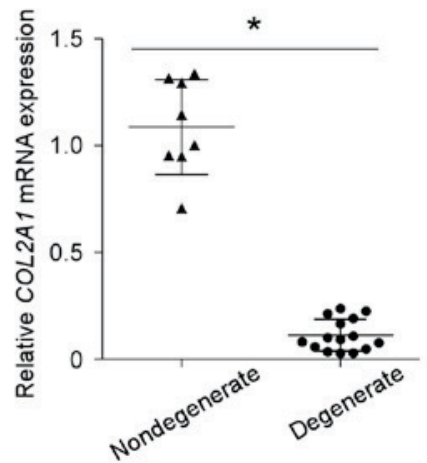

Figure 1. Expression of COL2A1 is downregulated in patients with DDD. (A) The expression of COL2A1 protein in 15 degenerated NP tissues and 8 nondegenerated NP tissues was detected by IHC. Representative images are presented at the indicated magnifications. Scale bar, $100 \mu \mathrm{m}$. (B) Semi-quantitative analysis of COL2A1 protein expression levels in tissues as measured by IHC. (C) COL2A1 mRNA expression levels were detected by reverse transcription-quantitative polymerase chain reaction. "P $<0.05$. COL2A1, collagen type II $\alpha 1$; DDD, degenerative disc disease; NP, nucleus pulposus; IHC, immunohistochemistry; IOD, integrated optical density.

A

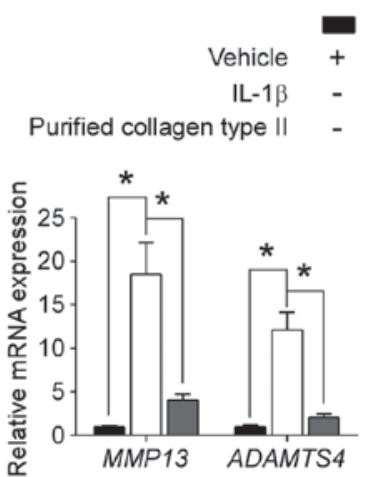

-

$+$

$+$

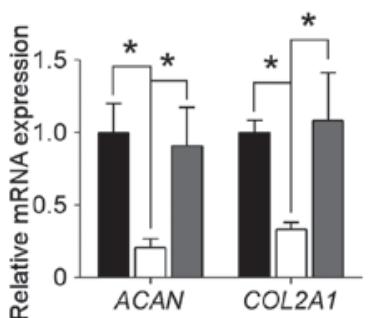

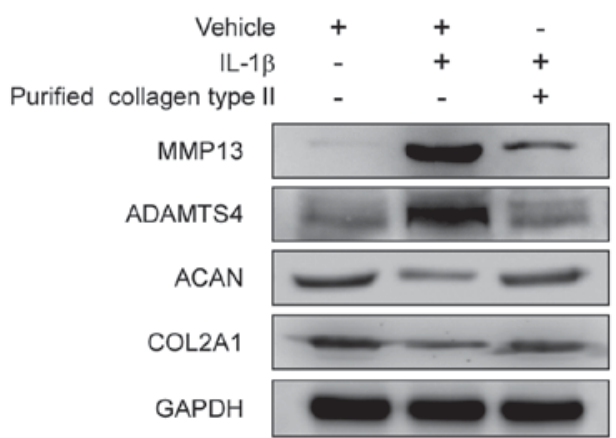

Figure 2. Collagen type II protects human NP cells from IL-1 $\beta$-induced metabolic disturbance. Human NP cells were treated with or without vehicle (0.05 M acetic acid), $10 \mathrm{ng} / \mathrm{ml} \mathrm{IL-1 \beta}$ and/or $100 \mu \mathrm{g} / \mathrm{ml}$ purified collagen type II for $48 \mathrm{~h}$, as indicated. (A) mRNA and (B) protein expression levels of MMP13, ADAMTS4, ACAN, and COL2A1 were detected by reverse transcription-quantitative polymerase chain reaction and western blotting, respectively. Data are presented as means \pm standard deviation of three independent experiments. ${ }^{*} \mathrm{P}<0.05$, with comparisons indicated by brackets. NP, nucleus pulposus; IL, interleukin; MMP13, matrix metalloproteinase 13; ADAMTS4, a disintegrin and metalloproteinase with thrombospondin motifs 4; ACAN, aggrecan; COL2A1, collagen type II $\alpha 1$.

effects on the IL-1 $\beta$-induced degenerative process of NP cells in vitro. The present findings therefore suggest that collagen type II may be a promising therapeutic target for the prevention and treatment of DDD.
NP cells synthesize and secrete cartilage-like ECM components, such as collagen type II and aggrecan, which are essential to NP homeostasis. In the course of NP degeneration, a decline in the number of NP cells, caused by apoptosis, 
A

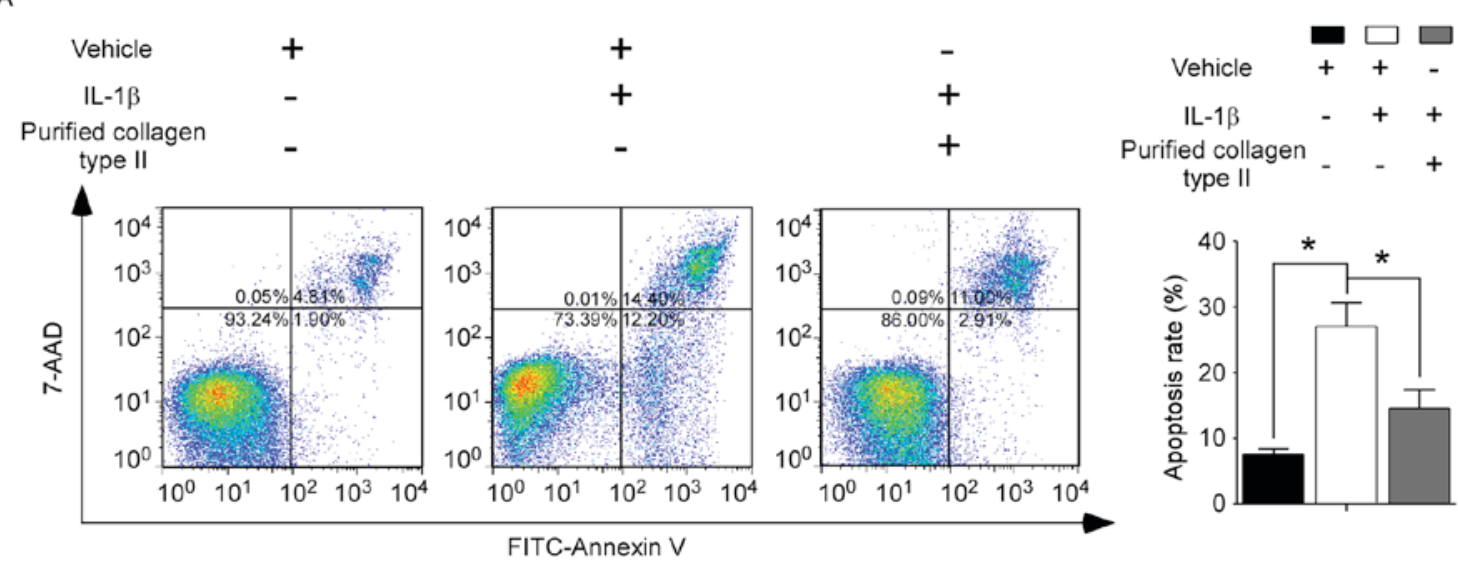

B

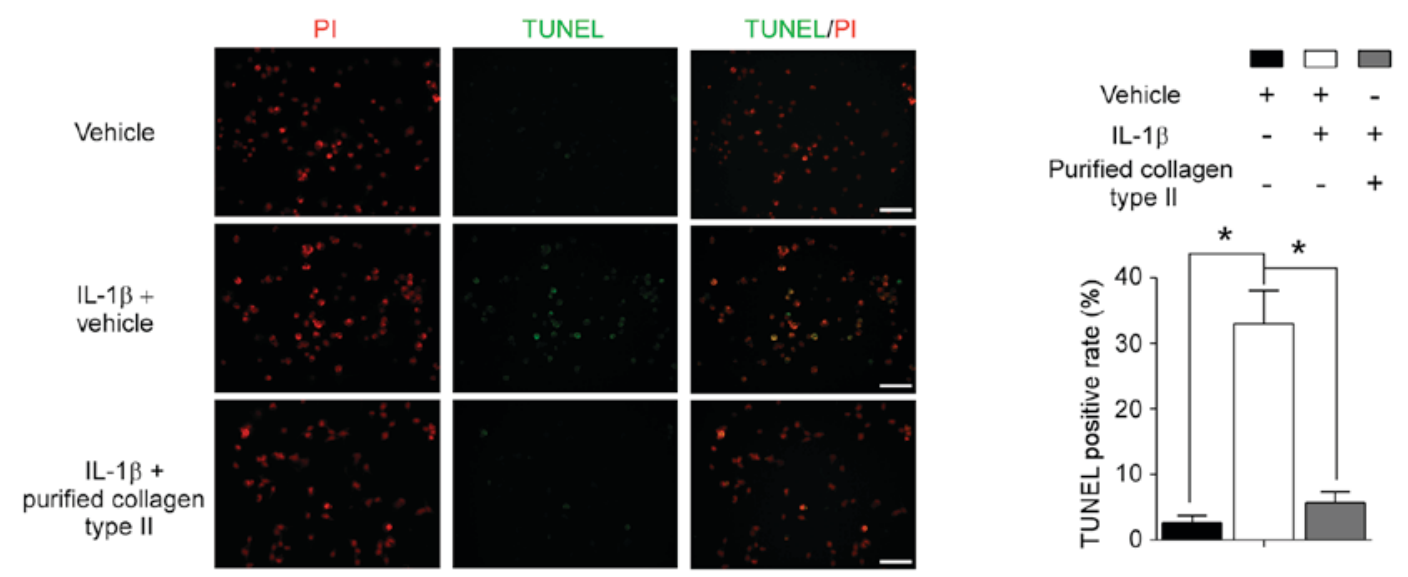

C

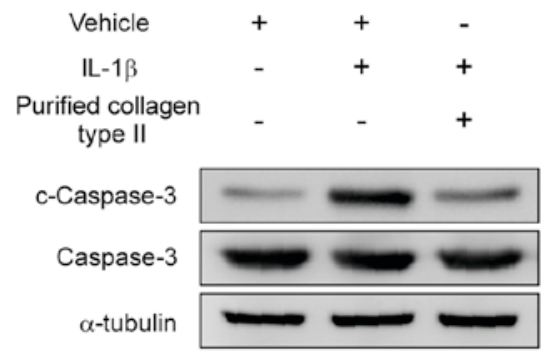

Figure 3. Collagen type II protects human NP cells from IL-1 $\beta$-induced apoptosis. Human NP cells were treated with or without vehicle (0.05 M acetic acid), $10 \mathrm{ng} / \mathrm{ml} \mathrm{IL}-1 \beta$ and/or $100 \mu \mathrm{g} / \mathrm{ml}$ purified collagen type II for $48 \mathrm{~h}$, as indicated. (A) Cells were stained with FITC-conjugated Annexin V and 7-AAD and analyzed by flow cytometry. Representative plots and quantification are shown as means \pm standard deviation of three independent experiments. (B) TUNEL assay was evaluated by fluorescence microscopy. Green fluorescence indicates apoptotic TUNEL-positive cells, while red fluorescence indicates PI-stained nuclei. Representative images and quantification are shown as means \pm standard deviation of three independent experiments. Scale bar, $100 \mu \mathrm{m}$. (C) Protein levels of c-caspase- 3 and total capase- 3 were evaluated by western blotting. " $\mathrm{P}<0.05$, with comparisons indicated by brackets. NP, nucleus pulposus; IL, interleukin; FITC, fluorescein isothiocyanate; 7-AAD, 7-aminoactinomycin D; TUNEL, terminal deoxynucleotidyl-transferase-mediated dUTP nick end labelling; PI, propidium iodide; c, cleaved.

leads to the reduced production of ECM. At the same time, increasing numbers of proinflammatory cytokines and catabolic proteases further degrade the existing ECM. The current study confirmed that, compared with the control group, both mRNA and protein levels of collagen type II were decreased in degenerating NP tissues. However, the discrepancy in the age of the DDD patients and the control group is a limitation of the present study. Human healthy nondegenerative NP tissues are rare and difficult to acquire, and patients with AIS are commonly used as nondegenerative controls $(16,17)$.
AIS usually occurs in adolescent females while DDD frequently occurs in elderly people, thus it is impossible to get age- and sex- matched controls. It is known that collagen fibrils decrease with age in NP tissues. The age difference between the two groups in the present study may influence the results, so age- and sex- matched nondegenerative NP tissues (possibly from patients with spinal fracture who require discectomy treatment) ought to be further collected and analyzed to verify the present findings. However, the half-life of collagen fibrils is long, being estimated to be 
more than decades (22). Therefore, under normal conditions, the decrease in collagen with age is limited, and the effect of age difference in the present study is unlikely to be the major cause for the decrease in collagen type II. The reduction of collagen type II is regarded as a hallmark of NP degeneration $(3,4)$. However, whether the lack of collagen type II can in turn influence the pathophysiological condition of NP cells remains unknown.

An increasing number of studies have reported that collagen type II can act as an extracellular signaling molecule that can regulate multiple biological functions in chondrocytes and mesenchymal stem cells (MSCs) (12-14,23). Chiu et al (14) reported that collagen type II promotes anabolism and maintains the cartilaginous phenotype of chondrocytes. In addition, collagen type II can regulate the differentiation of chondrocytes and MSCs (13,23). Klatt et al (12) demonstrated that collagen type II can modulate the inflammatory responses of chondrocytes. However, whether collagen type II can affect the biological functions of NP cells remains unknown. In the present study, IL-1 $\beta$ was used to induce the degeneration of human NP cells, as described previously $(8,24)$. IL- $1 \beta$ treatment alone upregulated the matrix degrading enzymes MMP13 and ADAMTS4, but downregulated the NP-characteristic ECM components aggrecan and collagen type II, in accordance with previous reports $(8,25)$. In the present study, addition of collagen type II to IL-1 $\beta$ treatment significantly reversed the IL-1 $\beta$-induced increase in catabolic genes and decrease in ECM genes, suggesting that collagen type II may have a pivotal role in maintaining the metabolic balance of NP cells. In addition, IL-1 $\beta$ induced apoptosis in NP cells, and treatment with collagen type II significantly protected against the IL- $1 \beta$-enhanced apoptosis of NP cells, thus potentially preventing the loss of NP cells and reduced production of ECM. Disordered ECM metabolism and excessive decrease in NP cells are regarded as the predominant pathological changes in degenerative IVDs $(5,20,24)$. Consequently, inhibition of the apoptosis of NP cells and ECM degradation is hypothesized to delay the progression of DDD. The present study demonstrated that collagen type II had anticatabolic, proanabolic, and antiapoptotic effects in human NP cells, therefore indicating that it may be a promising biological agent to promote the regeneration and repair of the degenerating NP.

The results of the present study demonstrated that collagen type II expression was decreased in the NP of patients with DDD. The reduced production of collagen type II may aggravate the metabolic disturbance, promote the apoptosis of NP cells, and facilitate continuing degeneration, which may subsequently result in a further reduction of collagen type II. The breakage of this vicious cycle is key to the treatment of DDD. Current therapies are still restricted to symptomatic treatment and do not correct or reverse the structural and biological deteriorations of the IVD. However, extensive studies indicate that biological therapeutic strategies, including cell transplantation, the use of biomaterial and gene therapy, may have broad applications $(3,4,7,21,26-28)$. Cell-based transplantation with biological enhancement is particularly important among these novel biological treatment approaches, because the functionality of NP cells is thought to be more important than the number of NP cells $(26,27)$.
Collagen type II, which can act as both structural matrix and bioactive factor for transplanted cells, may be an ideal agent for biological enhancement. Thus, intradiscal injection of suitable cells (such as NP cells, and undifferentiated, chondrogenic-like or NP-like differentiated MSCs) together with collagen type II, or injection of cells engineered to express the COL $2 \mathrm{~A} 1$ gene, may represent a promising therapeutic approach. This type of therapy would not only repopulate the NP with functional cells, but also create a beneficial microenvironment for NP cells by stimulating anabolism, inhibiting catabolism, and blocking apoptosis. Revealing the molecular mechanisms by which collagen type II modulates NP cell metabolism and facilitates NP cell survival is the focus of work that is underway in our group. Further studies will be needed in the future to confirm the therapeutic value, application conditions and safety issues involving the use of collagen type II in DDD treatment.

\section{Acknowledgements}

The present study was supported by the National Natural Science Foundation of China (grant nos. 81371907 and 81572134) and the Natural Science Foundation of Guangdong Province, China (grant no. 2016A030313284). We thank LetPub (www.letpub.com) for linguistic assistance during the preparation of this manuscript.

\section{References}

1. Hoy D, Bain C, Williams G, March L, Brooks P, Blyth F, Woolf A, Vos T and Buchbinder R: A systematic review of the global prevalence of low back pain. Arthritis Rheum 64: 2028-2037, 2012

2. Vos T, Flaxman AD, Naghavi M, Lozano R, Michaud C, Ezzati M, Shibuya K, Salomon JA, Abdalla S, Aboyans V, et al: Years lived with disability (YLDs) for 1160 sequelae of 289 diseases and injuries 1990-2010: A systematic analysis for the global burden of disease study 2010. Lancet 380: 2163-2196, 2012.

3. Colombier P, Clouet J, Hamel O, Lescaudron L and Guicheux J: The lumbar intervertebral disc: From embryonic development to degeneration. Joint Bone Spine 81: 125-129, 2014.

4. Fontana G, See E and Pandit A: Current trends in biologics delivery to restore intervertebral disc anabolism. Adv Drug Deliv Rev 84: 146-158, 2015.

5. Vo NV, Hartman RA, Yurube T, Jacobs LJ, Sowa GA and Kang JD: Expression and regulation of metalloproteinases and their inhibitors in intervertebral disc aging and degeneration. Spine J 13: 331-341, 2013.

6. Roberts S, Evans H, Trivedi J and Menage J: Histology and pathology of the human intervertebral disc. J Bone Joint Surg Am 88 (Suppl 2): S10-S14, 2006.

7. Sakai D and Grad S: Advancing the cellular and molecular therapy for intervertebral disc disease. Adv Drug Deliv Rev 84: 159-171, 2015.

8. Tran CM, Schoepflin ZR, Markova DZ, Kepler CK, Anderson DG, Shapiro IM and Risbud MV: CCN2 suppresses catabolic effects of interleukin-1 $\beta$ through $\alpha 5 \beta 1$ and $\alpha \mathrm{V} \beta 3$ integrins in nucleus pulposus cells: Implications in intervertebral disc degeneration. J Biol Chem 289: 7374-7387, 2014.

9. Kerr GJ, Veras MA, Kim MK and Séguin CA: Decoding the intervertebral disc: Unravelling the complexities of cell phenotypes and pathways associated with degeneration and mechanotransduction. Semin Cell Dev Biol 62: 94-103, 2017.

10. Deng H, Huang $X$ and Yuan L: Molecular genetics of the COL2A1-related disorders. Mutat Res Rev Mutat Res 768: 1-13, 2016.

11. Aszódi A, Chan D, Hunziker E, Bateman JF and Fässler R: Collagen II is essential for the removal of the notochord and the formation of intervertebral discs. J Cell Biol 143: 1399-1412, 1998. 
12. Klatt AR, Zech D, Kühn G, Paul-Klausch B, Klinger G, Renno JH, Schmidt J, Malchau G and Wielckens K: Discoidin domain receptor 2 mediates the collagen II-dependent release of interleukin-6 in primary human chondrocytes. J Pathol 218: 241-247, 2009.

13. Xin W, Heilig J, Paulsson M and Zaucke F: Collagen II regulates chondroycte integrin expression profile and differentiation. Connect Tissue Res 56: 307-314, 2015.

14. Chiu LH, Chen SC, Wu KC, Yang CB, Fang CL, Lai WF and Tsai YH: Differential effect of ECM molecules on re-expression of cartilaginous markers in near quiescent human chondrocytes. J Cell Physiol 226: 1981-1988, 2011.

15. Livak KJ and Schmittgen TD: Analysis of relative gene expression data using real-time quantitative PCR and the 2(-Delta Delta C(T)) method. Methods 25: 402-408, 2001.

16. Wang B, Wang D, Yan T and Yuan H: MiR-138-5p promotes TNF- $\alpha$-induced apoptosis in human intervertebral disc degeneration by targeting SIRT1 through PTEN/PI3K/Akt signaling. Exp Cell Res 345: 199-205, 2016.

17. Tsai TT, Lai PL, Liao JC, Fu TS, Niu CC, Chen LH, Lee MS, Chen WJ, Fang HC, Ho NY and Pang JH: Increased periostin gene expression in degenerative intervertebral disc cells. Spine J 13: 289-298, 2013.

18. Pfirrmann CW, Metzdorf A, Zanetti M, Hodler J and Boos N: Magnetic resonance classification of lumbar intervertebral disc degeneration. Spine (Phila Pa 1976) 26: 1873-1878, 2001

19. Boos N, Weissbach S, Rohrbach H, Weiler C, Spratt KF and Nerlich AG: Classification of age-related changes in lumbar intervertebral discs: 2002 Volvo Award in basic science. Spine (Phila Pa 1976) 27: 2631-2644, 2002.

20. Jiang W, Zhang X, Hao J, Shen J, Fang J, Dong W, Wang D, Zhang X, Shui W, Luo Y, et al: SIRT1 protects against apoptosis by promoting autophagy in degenerative human disc nucleus pulposus cells. Sci Rep 4: 7456, 2014.
21. Wang SZ, Chang Q, Lu J and Wang C: Growth factors and platelet-rich plasma: Promising biological strategies for early intervertebral disc degeneration. Int Orthop 39: 927-934, 2015.

22. Aigner T and Stöve J: Collagens-major component of the physiological cartilage matrix, major target of cartilage degeneration, major tool in cartilage repair. Adv Drug Deliv Rev 55: 1569-1593, 2003.

23. Chiu LH, Yeh TS, Huang HM, Lu SJ, Yang CB and Tsai YH Diverse effects of type II collagen on osteogenic and adipogenic differentiation of mesenchymal stem cells. J Cell Physiol 227: 2412-2420, 2012.

24. Hu B, Shi C, Xu C, Cao P, Tian Y, Zhang Y, Deng L, Chen H and Yuan W: Heme oxygenase-1 attenuates IL-1 $\beta$ induced alteration of anabolic and catabolic activities in intervertebral disc degeneration. Sci Rep 6: 21190, 2016.

25. Phillips KL, Jordan-Mahy N, Nicklin MJ and Le Maitre CL: Interleukin-1 receptor antagonist deficient mice provide insights into pathogenesis of human intervertebral disc degeneration. Ann Rheum Dis 72: 1860-1867, 2013.

26. Mern DS, Beierfuß A, Thomé C and Hegewald AA: Enhancing human nucleus pulposus cells for biological treatment approaches of degenerative intervertebral disc diseases: A systematic review. J Tissue Eng Regen Med 8: 925-936, 2014

27. Hou Y, Shi G, Shi J, Xu G, Guo Y and Xu P: Study design: In vitro and in vivo assessment of bone morphogenic protein 2 combined with platelet-rich plasma on treatment of disc degeneration. Int Orthop 40: 1143-1155, 2016.

28. Zhou X, Tao Y, Liang C, Zhang Y, Li H and Chen Q: BMP3 alone and together with TGF- $\beta$ promote the differentiation of human mesenchymal stem cells into a nucleus pulposus-like phenotype. Int J Mol Sci 16: 20344-20359, 2015. 